\title{
Experiencia vivida en madres/padres cuidadores de niños/niñas con Trastorno del Espectro Autista
}

\author{
Laura Gabriela Ráudez Chiong ${ }^{1}$ \\ Lisseth Carolina Rizo Aráuz ${ }^{2}$ \\ Franklin Jesús Solís Zúniga ${ }^{3}$
}

\section{RESUMEN}

Las vivencias de las madres/padres cuidadores de niños/niñas con trastorno del espectro autista, es poco conocida en Nicaragua, este tema ha estado en el anonimato y solamente las personas cercanas a un caso de autismo comprenden lo que significa el cuido de estos niños/niñas. De tal manera que el presente estudio se planteó como objetivo comprender la experiencia vivida de las madres/ padres cuidadores de niños/niñas con trastorno del espectro autista, en la ciudad de Estelí, segundo semestre 2016. El tipo de estudio es cualitativo y fenomenológico de tipo exploratorio, la muestra se conformó por un padre y cuatro madres cuidadoras de niños/niñas diagnosticadas con trastorno del espectro autista; y cinco expertas que trabajan con los mismos. Éstos fueron seleccionados mediante la técnica de cadena o bola de nieve. El tipo de muestreo es no probabilístico y por conveniencia. Los métodos teóricos que se utilizaron son el inductivo, método de análisis-síntesis; y los métodos empíricos fueron la entrevista a profundidad y la entrevista semi-estructurada. Los resultados más relevantes que se obtuvieron en cuanto a las vivencias, es que, en su totalidad las madres/padres cuidadores al momento de enterarse del diagnóstico tienen reacciones similares como, confusión, tristeza, pérdida, culpa, dolor, depresión, negación, impotencia, desesperación, baja autoestima y preocupación. Se logró comprender que la experiencia vivida de las madres/ padres cuidadores, radica principalmente en atravesar las etapas del duelo, ya que, en todos los casos, según los relatos obtenidos, se enfrentan al sentimiento de pérdida, como si su hijo/hija ha muerto.

Palabras clave: Experiencia vivida, madres/padres cuidadores, trastorno del espectro autista, duelo, repercusiones psicosociales.

Recibido: 01 de febrero de 2017

Aceptado: 24 de febrero de 2017

1 Egresada de la carrera de Licenciatura en Psicología. UNAN-Managua-FAREM-Estelí. Correo electronico: gabychiong1@yahoo.com.ar

2 Egresada de la carrera de Licenciatura en Psicología. UNAN-Managua-FAREM-Estelí. Correo electronico: lrizoarauz@gmail.com

2 Psicólogo-Docente. Universidad Nacional Autónoma de Nicaragua. Facultad Regional Multidisciplinaria de Estelí. Master en Psicología, Atención Personalizada y Desarrollo Comunitario. UAB. España. Doctorando en Ciencias en Salud Pública. Universidad de Guadalajara. México. Correo electrónico: franksolis23@yahoo.com 


\title{
Experience lived in mothers/fathers carers of children with Autism Spectrum Disorder
}

\begin{abstract}
The experiences of mothers/fathers carers of children with autism spectrum disorder is little known in Nicaragua, this issue has been anonymous and only people close to a case of autism understand what it means to care for these children. In such a way that the present study aimed to understand the lived experience of mothers/fathers carers of children with autism spectrum disorder, in the city of Esteli, second semester 2016. The type of study is qualitative and phenomenological of Exploratory type, the sample was formed by a father and four caregivers of children diagnosed with autism spectrum disorder; And five experts working with them. These were selected using the string or snowball technique. The type of sampling is non-probabilistic and for convenience. The theoretical methods that were used are the inductive, method of analysis-synthesis; And the empirical methods were the in-depth interview and the semi-structured interview. The most relevant results obtained in terms of experiences are that, in their entirety, the mothers/parents caregivers at the time of learning the diagnosis have similar reactions as confusion, sadness, loss, guilt, pain, depression, denial, impotence, Despair, low self-esteem and concern. It was possible to understand that the lived experience of the caretaker mothers/parents is mainly to go through the stages of mourning, since in all cases, according to the stories obtained, they face the loss feeling, as if their son/daughter has dead.
\end{abstract}

Keywords: Experience lived, mothers/fathers carers, autistic spectrum disorder, mourning, psychosocial repercussions. 


\section{INTRODUCCIÓN}

"Es un hecho universalmente aceptado que la presencia en las familias de un hijo con algún tipo de minusvalía física o psíquica grave constituye un factor potencial que perturba la dinámica familiar" (Martínez \& Bilbao, 2008).

La presente investigación, se realizó con el principal objetivo de comprender la experiencia vivida de las madres/padres cuidadores de niños/niñas con trastorno del espectro autista, en la ciudad de Estelí, segundo semestre del año 2016, se planteó investigar las vivencias de las madres/padres cuidadores de niños/niñas con espectro autista, las repercusiones psicosociales del rol que ejercen las madres/padres cuidadores en su calidad de vida, las estrategias de afrontamiento que utilizan las madres/padres cuidadores para sobrellevar el proceso del espectro autista.

Medio siglo después que el psiquiatra Leo Kanner (1943) describiera el "autismo", los avances de la psicopatología en este campo han sido relativamente pequeños. Hoy para la mayoría de los autores sigue siendo un misterio, o como Uta Frith (1991) reconoce en su obra, un enigma aún por resolver. (Belloch, Sandín, \& Ramos, 1995, pág. 652)

Los estudios encontrados reflejan que en las madres cuidadoras surgen reacciones como el duelo, la rabia al sentir la falta de comprensión por parte de personas que no son conscientes de las discapacidades de sus hijos, esto referido al mundo externo y no propiamente tal al trastorno autista que padece su hijo o hija, este estudio tiene como título "Experiencias de Madres de Niños o Niñas Diagnosticados Autistas en Estudio de Casos, desde una mirada Analítico Existencial". (Carolina Tobar Concha, 2014, pág. 51)

En el estudio "El impacto del autismo en la familia: Mi hijo después de la crisis" muestra que en todas las familias reinó la tristeza ante la noticia del impedimento.
De alguna forma $\mathrm{u}$ otra, todas las participantes pasaron por etapas que se asocian al duelo. Éstas son tristeza, rabia, negación, soledad y aceptación. Todas las participantes indican que son ellas las que toman las decisiones fuertes y que los otros miembros de la familia, al no entender las características que trae el espectro autista, piensan que son changuerías de los niños y los consienten de forma que si ellas no intervienen, el proceso de modificación de conducta se ve afectado (Rodríguez Carrión, 2012, pág. 147)

El estudio "Interpretación de emociones en madres de niños con trastorno del espectro autista", refiere "Así, se entendería el predominio de sentimientos de rabia y frustración que atenúen y contrarresten sus sentimientos de tristeza asociados a las dificultades que presentan sus hijos con TEA" (Castellanos, 2012).

Tomando en cuenta los estudios realizados anteriormente, esta investigación pretendía saber cuáles son las experiencias vividas de las madres/ padres cuidadores de niños/ niñas con trastorno del espectro autista para que se conozca desde esta perspectiva crucial y se puedan brindar estrategias de afrontamiento, así como también las de intervención psicológica para dichas personas, ya que son los principales afectados, además del niño o niña.

El estudio puede dar un aporte metodológico a cualquier equipo de profesionales que desee realizar un estudio similar o profundizar en el mismo, en tanto que detalla paso a paso la metodología del trabajo, la creación de métodos empíricos para la recolección de la información y el análisis de la misma.

La novedad de esta investigación se establece en que es la primera vez que se realiza un estudio de esta naturaleza en Nicaragua, enfocada en la experiencia vivida de madres/padres cuidadores de niños/niñas con trastorno del espectro autista, éste sector es muy importante ya que son los que están en contacto directo con éstos niños y niñas y son los que van a mejorar su calidad de vida. 


\section{MATERIALES Y MÉTODOS}

\section{Tipo de estudio}

El estudio utilizó la recolección de datos sin medición numérica para descubrir o afinar preguntas de investigación en el proceso de interpretación por lo cual es un estudio cualitativo y fenomenológico ya que estudia la vivencia del ser humano en una determinada situación procura interrogar la experiencia vivida de las madres/padres cuidadores de niños/niñas con trastorno del espectro autista, los significados que el sujeto le atribuye, así mismo conforme a lo que explican tanto Husserl como Heidegger se define a la fenomenología como el estudio de los fenómenos (o experiencias) tal como se presentan y la manera en que se vive por las propias personas, de la misma forma se partió principalmente de la experiencia y la percepción de las protagonistas a través de preguntas abiertas y así como también a los expertos que trabajan con el espectro autista. (Hernández, Fernández, \& Baptista, 2010).

El tipo de estudio que se realizó es exploratorio ya que se investigó un problema poco estudiado nacional e internacionalmente y se indagó desde una perspectiva innovadora, además se trabajó en un período de seis meses del segundo semestre del año 2016. (Hernández, Fernández, \& Baptista, 2010).

\section{Sujetos participantes}

Se trabajó con una muestra conformada por cuatro madres y un padre cuidadores de niños/niñas diagnosticados con trastorno del espectro autista $\mathrm{y}$ cinco expertos que trabajan con este tipo de niños/ niñas.

\section{Métodos empíricos}

Los relatos fueron obtenidos a través de una entrevista a profundidad aplicada a las madres/padres cuidadores y una entrevista semiestructurada aplicada a las expertas en el tema.

\section{Tipo de muestreo}

Las madres/padres cuidadores y expertos fueron seleccionados a través de la técnica de cadenas o bola de nieve ya que se identificaron participantes claves y se agregaron, a los mismos se les preguntó si conocían a otras personas que tuvieran las mismas características y puedan proporcionar datos acerca del tema, de tal manera que no se trabajó con ningún centro de atención específico.

El tipo de muestreo es no probabilístico, ya que se seleccionó a los participantes por un propósito específico y no pretendía que la muestra fuese representativa a toda la población (Hernández, Fernández, \& Baptista, 2010), se utilizó el método de muestreo por conveniencia el cual consiste en seleccionar a los individuos que le convienen al investigador para la muestra. Esta conveniencia se produce porque al investigador le resulta más fácil examinar a estos sujetos, ya sea por proximidad geográfica, por ser sus amigos, además el procedimiento no se hizo con base a fórmulas de probabilidad sino que dependía del proceso de la toma de decisiones del investigador. Por lo cual se definieron criterios para la selección de la muestra.

\section{Criterios de selección}

Para optar a formar parte de la investigación, los participantes, debían cumplir ciertos criterios o características con base al tema de investigación:

- Personas que sean madres/padres cuidadores de niños/niñas con trastorno del espectro autista.

- Qué pertenezcan al Municipio de Estelí

- Disponibilidad para participar en el estudio y compartir sus experiencias así como también ayudar a describirlas.

Además se eligieron a expertos en el tema para profundizar los conocimientos acerca del trastorno y las estrategias de intervención psicológica, estos expertos debían cumplir con los siguientes criterios: 
- Expertos que sean psicólogos o psiquiatras y médicos.

- Expertos que hayan trabajado con niños/niñas que presenten el trastorno espectro autista.

- Disponibilidad de participar en el estudio.

- Que pertenezcan al Municipio de Estelí.

\section{Procesamiento y análisis de datos}

El procesamiento y análisis de datos se realizó en función de los objetivos formulados a través de una matriz de transcripción y análisis, se transcribieron las respuestas de las protagonistas fielmente para su respectivo análisis.

\section{Aspectos éticos}

Para darle autenticidad al estudio, se realizó un consentimiento informado en donde se detallan los aspectos éticos de esta investigación. Ahí se explica a las/los protagonistas en qué consiste su participación, el propósito del estudio, la privacidad y confidencialidad, ésta refleja que los datos obtenidos permanecerán en confidencialidad de las investigadoras, además que pueden ser publicados en revistas científicas o ser presentados en reuniones científicas, pero, la identidad de las/los protagonistas no será divulgada.

El derecho a retirarse del estudio, sin embargo, se aclara que los datos obtenidos serán utilizados para el estudio y que al retirar su participación deben informar a las investigadoras. Al final del consentimiento se detallan solamente las iniciales y firma del participante respetando de esta forma su identidad.

\section{RESULTADOS Y DISCUSIÓN}

\section{Vivencias de las madres/padres cuidadores}

De acuerdo al análisis que se realizó de los datos obtenidos se observa que cada una de las madres/ padres, al recibir el diagnóstico del trastorno del espectro autista en sus hijos/hijas, tienen sentimientos y reacciones similares como, confusión al no saber de lo que se trata el trastorno, tristeza, dejaron de trazar metas, pérdida, culpa, dolor, depresión, negación, desesperación, impotencia, baja autoestima y preocupación.

"Sentís una sensación de vacio como que algo te quitaron, que ya tenías y que te quitaron, culpa, porque te empezás a analizar desde que saliste embarazada, durante todo el embarazo, durante el parto o cesárea, durante las primeras etapas, es una situación como un duelo, es un proceso que hay que vivir porque es que te quitaron, a míme quitaron, se me quitó a mi hijo el hijo que yo tuve, y ahora le toca a uno aceptar poco a poco esta nueva persona que es completamente diferente y que tienen muchas dificultades para expresarse". (Madre 1)

"Básicamente no sabíamos mucho pero como nosotros teníamos la experiencia con el primer niño pues vimos raro que el último no hablara y otras cosas y por eso mi esposo investigó”. (Madre 5)

"Es como cuando se te muere alguien, los padres viven un duelo, cuesta que salgan de eso y aceptar, mientras ellos están viviendo ese duelo los niños no avanzan, como que no aceptan ese diagnóstico entonces se aferran, los niños perciben y tampoco dan espacio para avanzar" (Experta 5)

Las expertas entrevistadas coinciden en que una de las principales reacciones es la negación, las madres/ padres cuidadores no aceptan en primera instancia que su hijo/hija presenta este trastorno.

Cabe señalar que dos de las expertas entrevistadas expresan que cuando existe negación de las madres/ padres cuidadores, el niño/niña no presenta avances en las terapias, pero, cuando el diagnóstico es aceptado internamente o emocionalmente, el niño/niña empieza a mostrar cambios representativos. 
"la barrera de no querer aceptar que hay algo, pero también hemos visto que cuando uno como mama, como familia aceptan que hay algo, inmediatamente ves el cambio de los niños, no porque acepten que hay una discapacidad, ni porque lo van a tratar diferente sino que internamente, emocionalmente cambia algo", (Experta 3)

En cuanto a los familiares de las madres/padres cuidadores de los niños/as, la mayoría de ellos también ha pasado por la negación, el dolor, la confusión por no saber de qué trata el trastorno.

Lo han ido superando poco a poco, aunque aún hay familias en las que algunos miembros aceptan al niño/a pero no al diagnóstico y creen que es mal comportamiento o mala educación por parte de las madres/padres.

"Al principio la negación, en casi toda la familia a excepción de mi mama, mi suegra todavía no ha llegado a ese punto y no creo que llegue porque se niega completamente a asistir a un grupo, a una reunión, cualquier cosa que tenga que ver con autismo. Ella lo acepta, lo adora, pero no está en la parte en la que ella me pueda servir como apoyo para ayudar al niño”. (Madre 1)

"La niña se ha recibido con mucho amor en esta casa, porque mi papá un día me dijo "yo antes no entendía que era autismo y ahora el leer e informarme de esto y convivir con la niña me ha ayudado a comprender que es autismo" (Madre 2)

Otra de las vivencias son las dificultades por las que las madres/padres de niños y niñas con TEA deben enfrentar es la de no tener centros especializados en atención de estos niños/niñas.

"no hay en Nicaragua, centros especializados donde los profesores manejen este tema y cuiden a los niños, es decir, que comprendan a los niños que están ahí que tienen ese problema y que no se asusten, porque a veces se asustan cuando empiezan a hacer berrinches, que a veces les da agresividad, que les da hiperactividad, todas esas cosas no lo comprenden los maestros" (Padre)

Además, el desconocimiento que tiene la sociedad acerca del trastorno porque esto provoca según los relatos de ambas fuentes discriminación, esto hace que las personas vean a los niños/a con espectro autista con mala conducta o como popularmente los llaman "malcriados", arrogantes o prepotentes ya que ellos actúan de una manera agresiva cuando se sienten en un lugar desconocido y su forma de expresarse no es convencional.

"La sociedad yo creo que está por decir así verde, está todavía en etapas muy atrasadas de este conocimiento, la sociedad no domina absolutamente nada, si hablamos de que la parte de los médicos no dominan este tipo de diagnóstico, mucho menos la sociedad en general, no dominan nada". (Padre)

\section{Repercusiones psicosociales del rol que ejercen las madres/padres cuidadores de niños/niñas con trastorno del espectro autista}

"La situación de depresión y estrés puede aumentar como si fuera una lupa, pequeños problemas familiares, convirtiéndolos en dificultades serias de relación en la pareja. Complicarse, tomando la forma de un bucle de culpabilizaciones recíprocas. Dificultar la atención a los hermanos. Tener, en fin, consecuencias negativas de diversos órdenes en la economía de las relaciones familiares" (Martínez Martín \& Bilbao León , 2008)

El análisis de datos de este objetivo muestra que las consecuencias emocionales que les ha traído el diagnóstico a las madres/padres cuidadores radican, en la desesperanza y eliminación de metas, sueños y una familia que brinde atención proporcional a todos los miembros.

Angustia y temor permanente de que ocurra algo y las madres/padres cuidadores no se puedan enterar por la 
dificultad de comunicación que presentan los niños/as con trastorno del espectro autista. La impotencia y la presión de no saber lo que acontezca en el futuro, ya que en la mayoría de los casos no se sabe si se logrará una independencia total del niño/a.

"estrés constante y la angustia permanente, en el sentido de que, mi hijo va a la escuela y yo estoy con los ojos cerrados, porque no puedo ver, no sé qué le pasa a mi hijo y sólo me queda pedirle a Dios y confiar en las maestras de que está bien, pero es una angustia permanente todo el tiempo, mi mayor temor es que le pase algo" (Madre 1)

"Implica desgaste fisico y emocional de los padres, en lo fisico padecen de dolor de cabeza, cefalea tensional, problemas de insomnio, en lo emocional ansiedad, depresión, ya que no les cuidan a los niños por la sintomatología que presentan”. (Experta 1)

El desánimo que se presenta ante el reconocimiento del trastorno y la asimilación del cambio de estilo de vida. "la impotencia de no poder darle toda la atención que verdaderamente el necesite tal vez no estamos nosotros en la capacidad de darle lo que verdaderamente necesite para desarrollarse" (Madre 3)

Dificultad de procesar el duelo, ya que existe negación por el hecho de no tener la disponibilidad y la voluntad de documentarse acerca del trastorno. "La frustración es bien marcada, porque en medio del núcleo familiar es una lucha, tal vez el papa medio lo acepta y la mama no, o viceversa entonces es una lucha y es un conflicto" (Experta 3)

Estrategias de afrontamiento que utilizan las madres/ padres cuidadores para sobrellevar el proceso del trastorno del espectro autista

De acuerdo al análisis realizado las estrategias de afrontamiento que utilizan las madres/padres cuidadores de niños/niñas con trastorno del espectro autista son la unidad y comunicación como pareja, en cuanto a la división del trabajo con el niño/a, en los casos donde están juntos el papá y la mamá, el apoyo familiar no sólo de los padres sino de los abuelos, tíos, hermanitos, sobrinos.

Buscando apoyo en las organizaciones como los pipitos, que abordan el tema del trastorno del espectro autista, asistir a grupos de apoyo donde asisten madres/padres de niños/niñas con trastorno del espectro autista para retroalimentar la experiencia y utilizar estrategias de manejo en el cuido de los niños, además para fortalecer la autoestima y dar esperanza los unos a los otros.

Apoyo psicológico especializado en el tema para realizar sesiones de estimulación a los niños y niñas, Adaptarse a las necesidades que se van presentando. Búsqueda de apoyo en instituciones religiosas.

Búsqueda de especialistas como logopedas para la estimulación del lenguaje, así como también la búsqueda de un colegio donde exista personal calificado para la atención de niños y niñas TEA como Fundación Cristal ya que en algunos casos los padres no tienen tiempo disponible para realizar las terapias en casa.

"Algunas veces hemos visto programas en la tv, a veces se toca el tema en programas y eso le ayuda a la población a enterarse, el internet, pero la mejor manera ha sido el convivir con la niña, porque una cosa es leer, ver videos, escuchar testimonios, pero la mejor manera de darnos cuenta o informarnos es convivir con ella" (Madre 2)

"Por medio de internet o a través de casos, por lo menos por mi trabajo que es de bastante comunicación con la gente, porque uno de una u otra manera saca una plática, entonces, la gente me cuenta o ya les cuento yo lo que me pasa y así uno va a aprendiendo o los artículos que salen porque ahora publican bastante". (Madre 3) 
Estrategias de intervención psicológica que contribuyan al mejoramiento de la calidad de vida de las madres/padres cuidadores de niños/niñas con trastorno del espectro autista

Las estrategias de intervención psicológica que a continuación se presentan fueron construidas con el aporte importante de las expertas, así mismo, con la opinión de las madres /padres cuidadores, ya que, son ellos quienes están permanentemente con los niños/ niñas.

- Terapias psicológicas a madres/padres cuidadores para que se informen del trastorno y disminuyan el estrés que el cuido provoca.

- Crearles espacios de autoayuda y auto cuido, donde puedan relajarse y hablar del tema, para disminuir la carga que conlleva el cuido de estos niños.

- Crear espacios informativos sobre el cuido de niños/niñas TEA donde asista toda la familia, para que todos y todas miembros de la familia aporten.

- Dar atención psicológica a las madres/padres cuidadores enfocada en el duelo, para que las etapas sean superadas fácilmente.

- Crear espacios donde las madres/padres que ya pasaron por las etapas del duelo compartan su experiencia con las madres/padres que acaban de recibir el Diagnóstico.

- Crear espacios recreativos donde asistan las madres/padres cuidadores, familiares y los niños/ niñas que presentan el trastorno, en donde su relación sea meramente lúdica.

\section{CONCLUSIONES}

El cuidado de un niño autista resulta mucho más estresante para los padres que el cuidado de un niño con retraso mental (Dunn, Burbine, et al., 2001). Los padres de los niños autistas suelen encontrarse ante la frustración de intentar comprender a su hijo, de atenderlo día y noche, y buscar constantemente recursos educativos que le permitan avanzar en su desarrollo (Butcher, Mineka, \& Hooley, 2007)
El diagnóstico del trastorno del espectro autista en cualquier miembro de la familia es un impacto que cambia la dinámica de ésta, los relatos de las madres/ padres cuidadores permitieron describir su vivencia como cuidadores de niños/niñas con trastorno del espectro autista, la mayoría de las madres/padres cuidadores, pasan por las etapas de duelo, al sentir una pérdida, tal cual, si hubiera muerto un hijo, a raíz de recibir el diagnóstico, algunas manifestaron sentir "que les quitaron algo".

La presencia de un niño/niña con trastorno del espectro autista en el seno familiar, constituye una situación perturbadora que afecta a todos sus integrantes, ya que, es generada por el propio comportamiento del niño, también incluye alteraciones en la interacción social, déficit de comunicación y actividades e intereses restringidos, situación que se ve reflejada en las actitudes de las madres/padres cuidadores, provocando cambios en los estilos de vida de los mismos y afectando la dinámica familiar. (Hernández, Cárdenas, Zamalloa , \& Silva, 2012).

El hecho de no saber de qué trataba el trastorno fue un factor determinante para desestabilizar el sistema de emociones y principalmente introducirse en negación, culpabilidad e impotencia, sin embargo, esto incitó la activación para la búsqueda de información de dicho trastorno.

En la mayoría de los familiares de las madres/padres cuidadores también reinó la tristeza y la negación, aunque aceptaran al niño/niña, se niegan a aceptar el diagnóstico, incluso en algunos casos existe separación de la pareja matrimonial, esto causa que para las madres/padres cuidadores sea más difícil sobrellevar o enfrentar el proceso, ya que, no cuentan con el apoyo necesario, ni de familiares, ni de su pareja, para brindarle la mejor atención a su hijo/hija y que éstos logren un avance psicosocial significativo. 
De acuerdo al análisis realizado, los aportes de las expertas y los relatos de las protagonistas, se identificó que las repercusiones psicosociales del rol que ejercen las madres/padres cuidadores residen en la angustia y el temor permanente de lo que pueda ocurrir con su hijo/ hija fuera de casa, además, existe impotencia, presión e incertidumbre de lo que ocurrirá en el futuro con el niño/a, así mismo, dificultad de procesar el duelo.

De igual forma prevalece la frustración cuando se espera un desarrollo normal en el niño/niña y éste no lo presenta, el aislamiento, ya que, en algunos casos las madres/padres prefieren privarse de las actividades o eventos que se les presenta por no tener claro que reacción podría tener su hijo/hija y el estrés permanente por el cuido especializado que conllevan estos niños/ niñas.

Se identificó que las estrategias de afrontamiento utilizadas por las madres/padres cuidadores se establecen en la unidad y la comunicación en pareja, (en caso de que la tengan), búsqueda de apoyo en distintas organizaciones especializadas en el tema e instituciones religiosas, grupos de apoyo, soporte psicológico para realizar sesiones de estimulación con los niños/niñas, utilización de fuentes informativas como el internet, programas de televisión, videos, artículos, testimonios, intercambio de experiencias, entre otros.

Dentro de la investigación se establecieron propuestas de intervención psicológica que contribuyen al mejoramiento de la calidad de vida de las madres/padres cuidadores, éstas fueron elaboradas con los aportes de las expertas y la opinión de las/los protagonistas, se planteó la realización de terapias psicológicas a madres/ padres cuidadores para distintos fines (informativos, disminuir el estrés, duelo), la creación de espacios recreativos para madres/padres cuidadores, familiares y niños/niñas, creación de espacios de auto cuido para las madres/padres cuidadores, espacios informativos para los familiares sobre el cuido de los niños/as, grupos de autoayuda.
Se logró cumplir con todas las preguntas de investigación y objetivos planteados, se alcanzó comprender la experiencia vivida de madres/padres cuidadores de niños/niñas con trastorno del espectro autista, a través de la voz de las protagonistas, así como también de las expertas, los relatos fueron de vital importancia para el acercamiento con cada una de las vivencias.

\section{RECONOCIMIENTOS}

- A nuestros padres por el apoyo incondicional que nos han brindado a lo largo del camino,

- A nuestros esposos por acompañarnos en esta etapa de crecimiento personal,

- A nuestro tutor MS.c. Franklin Solís, por el seguimiento y por los conocimientos compartidos en el proceso.

- A nuestras familias por sus palabras de aliento y su presencia,

- A mis suegros por el soporte otorgado,

- Finalmente agradecemos a cada una de las expertas y protagonistas (madres/padre), que dieron su valioso tiempo para participar y compartir sus experiencias, ya que, sin sus relatos no hubiese sido posible la realización de este estudio.

\section{REFERENCIAS BIBLIOGRÁFICAS}

Belloch, A., Sandín, B., \& Ramos , F. (1995). Manual de Psicopatología Volumen 2. España: McGrawHill/Interamericana de España S.A.

Butcher, J. N., Mineka, S., \& Hooley, J. M. (2007). Psicología Clínica 12 Edición. Madrid: Pearson Educación S.A.

Carolina Tobar Concha, C. G. (Enero de 2014). Experiencias de Madres de Niños o Niñas Diagnosticados Autistas en Estudio de Casos, desde una mirada Analítico Existencial. Tesina para optar al grado de Licenciado en Psicología. Obtenido de http://bibliotecadigital.academia.cl/ bitstream/handle/123456789/1361/tpsico\%20559. 
pdf? sequence $=1$

Castellanos Flores, P. (2012). Interpretación de emociones en madres de niños con trastorno del espectro autista. Tesis para optar el título de Licenciada en Psicología con mención en Psicología Clínica. Obtenido de http://m.tesis.pucp.edu.pe/ repositorio/bitstream/handle/123456789/1725/ CASTELLANOS_FLORES_PAOLA_ INTERPRETACION_TRANSTORNO. pdf?sequence $=1 \&$ isAllowed $=\mathrm{y}$

Hernández , A., Cárdenas Gutiérrez, M., Pastor Zamalloa, M., \& Silva Mathews, Z. (2012). Experiencia de los padres ante el cuidado de su hijo autista. Obtenido de http://www.upch.edu. pe/faenf/images/pdf/Revistas/2012/enero/04\%20 padres $\% 20$ ante $\% 20$ el $\% 20$ cuidado $\% 20$ de $\% 20$ su\%20hijo\%20autista.pdf
Hernández Sampieri, R., Fernández Collado, C., \& Baptista Lucio, P. (2010). Metodología de la Investigación Quinta Edición. México: McGRAWHILL / INTERAMERICANA EDITORES, S.A. DE C.V.

Martínez Martín, M., \& Bilbao León , M. (9 de Nov de 2008). Acercamiento a la realidad de las familias de personas con autismo. Obtenido de Psychosocial Intervention vol.17 no.2 Madrid Obtenido de: http://scielo.isciii.es/scielo.php? script=sci_ arttext\&pid=S1132-05592008002200009

Rodríguez Carrión, M. (Mayo de 2012). Tesis, El impacto del trastorno del espectro autista en la familia:. Obtenido de http://www.suagm.edu/ utdoctoral/pdfs/Disertaciones-2012/MilagrosRodriguez-Tesis-2012.pdf 